\title{
Features of Impinging Streams Intensifying Processes and Their Applications
}

\author{
Yuan Wu, Yuxin Zhou, Jia Guo, and Jun Yuan \\ Key Laboratory for Green Chemical Processes of Education Ministry and Hubei Province, School of Chemical Engineering \& Pharmacy, \\ Wuhan Institute of Technology, Wuhan 430073, China \\ Correspondence should be addressed to Yuan Wu, wuyuan@public.wh.hb.cn
}

Received 12 August 2009; Revised 8 December 2009; Accepted 27 January 2010

Academic Editor: B. Kulkarni

Copyright ( $(2010$ Yuan Wu et al. This is an open access article distributed under the Creative Commons Attribution License, which permits unrestricted use, distribution, and reproduction in any medium, provided the original work is properly cited.

Impinging streams (IS) are classified into gas-continuous and liquid-continuous ones (GIS and LIS). Large number of experimental data has shown GIS promotes transfer very efficiently; while it has the intrinsic faultiness of very short residence time, and its flow configuration is relatively complex, resulting in difficulty in arranging multistage process. Essentially, GIS is applicable only for rapid processes controlled by gas film diffusion. The effect of LIS enhancing transfer is negligible; while it has the features of efficient micromixing and strong pressure fluctuation both which are resulted from the intensive interaction between the opposing streams and can promote process kinetics. All the features of IS, including GIS and LIS, have great potential of application. Reviewing the results of number investigations, mostly worked by the authors, a somewhat detailed introduction to the features of IS and several cases of its successful applications, such as wet FGD, preparation of ultrafine or nano powders, successful industrial application of large scale LIS reactors and crystallizers, etc, are described.

\section{Introduction}

Impinging streams (IS), as a novel technical method in chemical engineering, first presented by Elperin [1] was aimed at enhancement of transfer between solid and gas phases, as shown in Figure 1. Because of its well properties and application potential, wide investigations on IS were made in the former Soviet Union, Israel [2], China, and some other countries since 1960s; the concept of IS was also extended, including the extension of phase conditions, that is, the continuous phase in IS can also be a liquid. Results of number investigations have illustrated that the properties and performances of IS with a liquid as the continuous phases are quite different from those with a gas as the continuous phase [3]. In order to favor understanding, $\mathrm{Wu}$ $[4,5]$ proposed the supplemental classification, classifying IS into two categories: gas-continuous impinging streams (GIS) and liquid-continuous impinging streams (LIS). The features of both GIS and LIS have important values for application.

In addition, to make the concept clear, the definition of IS was proposed as follows [6]. Impinging Streams are the flow configuration in which two continuous steams with certain momentum fluxes, containing dispersed phase(s) or not, flowing along the opposing directions, respectively, vertical to the bisection across the impinging point, impinge against each other.

Reviewing the results from a number of researches and developments, mostly worked by the authors, the present paper introduces the major features of IS of the two categories and some of their remarkable application cases.

\section{Major Features of Gas-Continuous Impinging Streams}

In GIS heat and mass transfer are efficiently enhanced mainly by the following $[2-5,7]$ : (1) very high relative velocity between phases, especially around the impingement plane; (2) oscillation movement of particles or droplets penetrating to and fro between the opposing streams; (3) very strong turbulence in the impingement zone. Also, in the case of dispersed phase being a liquid, surface renewing promoted by the impingement may lead to additional enhancement of transfer. So many data obtained by various researchers have shown that heat and mass transfer coefficients in GIS can be higher than those in traditional devices by over 10 or, sometimes, even by several decade times. Huang et al. 


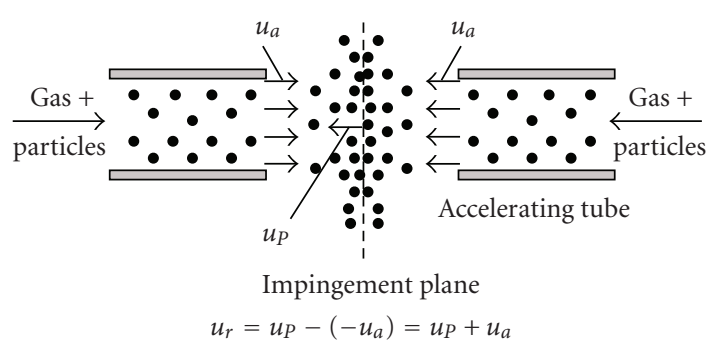

FIgURE 1: Original idea of impinging streams [2].

[8] and Wu et al. [9], in their investigations into circulative impinging stream drying (ISD) and desulfurization of flue gas in the impinging stream gas-liquid reactor (ISGLR), respectively, obtained newer experimental evidences The former showed the values for volumetric heat transfer coefficient in ISD being higher than in spray dryer by over 10 times; while the latter demonstrated the values of 0.58 to $1.04 \mathrm{~s}^{-1}$ for the same parameter in ISGLR, which are equivalent to or a little higher than those in rotary packed bed (RPB), another kind of well-known device also enhancing transfer very efficiently, and the detailed data have been reported in [9].

Another notable nature of GIS is strong collisions between particles in the impingement zone. In many cases, for example, when the particles are used as a catalyst, this property is harmful as it crushes particles and thus increases the loss of catalyst; while in the others strong collision can be used for crushing and grinding of solid materials. In fact grinding or milling of solids is a field where GIS earlier applied successfully in industries, and the well-known Trost Jet mill has been collected into the standard tool book [10] as early as in 1970s. Because it is not a very important application of IS, this topic will not be discussed further in the present paper.

On the other hand, GIS has the intrinsic faultiness of very short residence time in the active region, mainly the impingement zone; its flow configuration is relatively complex so that any arrangement of multistage GIS would be difficult and energy consuming. Therefore GIS alone is applicable essentially only for the processes involving rapid reaction(s) on surface of or inside the particles or droplets in dispersed phase and thus controlled by gas-film diffusion, such as powdery coal or sprayed liquid fuel burning and chemical absorption involving instant reaction in liquid, and so forth.

\section{Features of Liquid-Continuous Impinging Streams}

Liquid-continuous impinging streams (LIS) have been found to have the following major features of great significance for application.

3.1. Negligible Effect on Enhancement of Transfer. With LIS, because of the properties of liquid, mainly high density and viscosity, the factors enhancing transfer in GIS mentioned in the last section are essentially disappeared so that the effect of LIS enhancing transfer is negligible. Tamir et al. [11, 12] and Davies [13] provide experimental evidences, although the point is opposite to Tamir's opinion. The former reported the values of $1.33 \times 10^{-4}$ and $1.60 \times 10^{-4} \mathrm{~m} \cdot \mathrm{s}^{-1}$ for the mass transfer coefficients in dissolution of urea into water in two LIS devices of different structures; while the data obtained by Davies for the same parameter in dissolution of sodium chloride and potassium sulphate into water in the traditional stirred tank reactor were $1.0 \times 10^{-4}$ and $1.26 \mathrm{~m} \cdot \mathrm{s}^{-1}$, respectively. The difference between the physical properties of the substances involved seems favoring the dissolution of urea. From these data less enhancement of mass transfer can be observed.

On the other hand, high density of liquid leads to strong interaction between the opposing streams in LIS, resulting in a number of excellent features, as described below.

3.2. Efficient Micromixing. Micromixing is a critical condition for processes occurring on molecular scale in liquid phase. The interaction between opposing streams in LIS, including collisions, pressing, and shearing between fluid elements, reduces the segregation scale, and when the latter is down to the Kolmogoroff microscale, $\lambda$, molecular diffusion takes over to achieve complete homogeneity. Wu et al. [14] studied micromixing in the submerged circulative impinging stream reactor (SCISR), the first LIS reactor developed by Wu [15], shown in Figure 2, and obtained the following major results indicating very well micromixing nature of LIS. (1) The values for the characteristic micromixing time constant, or simply called micromixing time, $t_{M}$, determined in the impinging velocity range of $0.184<u_{0}<0.326 \mathrm{~m} \cdot \mathrm{s}^{-1}$ are ranged from 192 to 87 milliseconds and is less than 100 milliseconds at normal operating rotary speed of the propellers, where the term "impinging velocity" is defined as the stream velocity at the outlet of the drawing tubes, while, in comparison, the values measured in the traditional stirred tank reactor (STR) are usually in the range from 500 to 800 milliseconds; (2) the measured data meet about the relationship of $t_{M} \propto u_{0}^{-1.5}$; (3) the values for $t_{M}$ predicted with the existing model, which was established from the data obtained with STR, are systematically longer than those measured by about 2 to 3 times, implying that micromixing condition in SCISR is greatly better than predicted theoretically. Another notable finding in this study is that the Reynolds number from the $\pi$-law, Re, cannot be used as the criterion for scaling-up of impinging streams. Otherwise large-scale system must be operated at very low impinging velocity to keep the same value for $\mathrm{Re}$ as in 


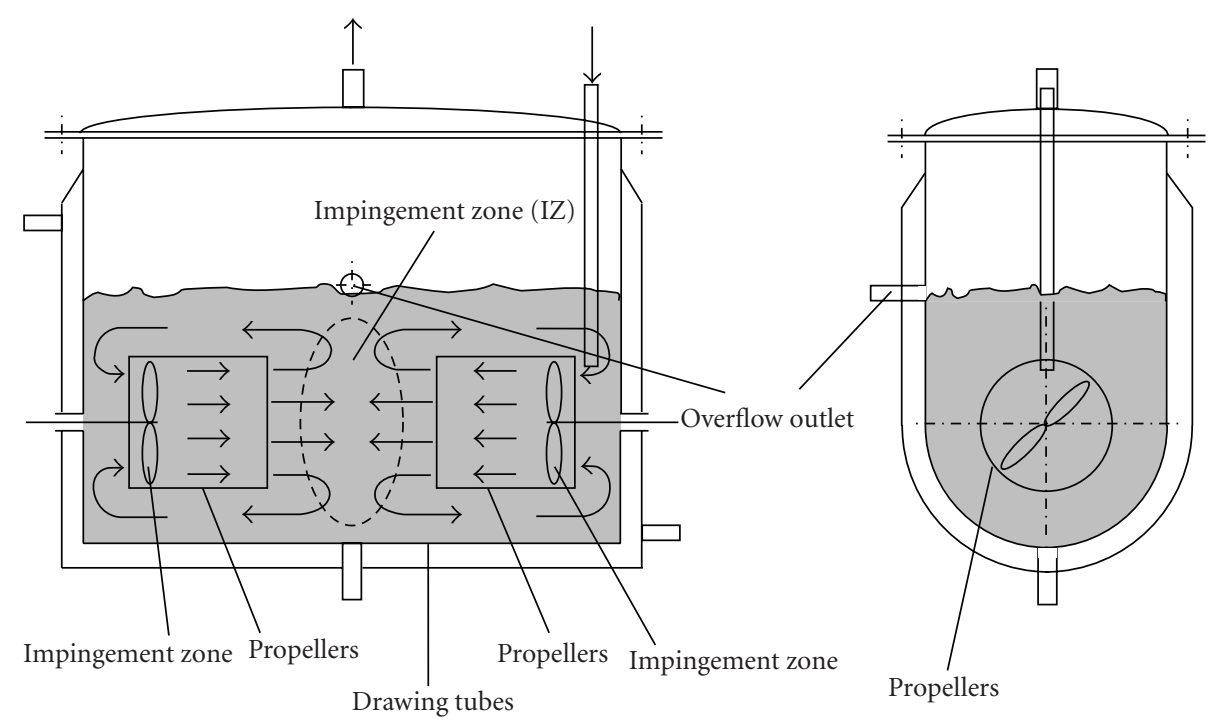

FIGURE 2: A brief view of SCISR 1-drawing tubes; 2-propellers; 3 impingement zone; 4-overflow outlet IZ-impingement zone.

small model device; while this implies directly very weak momentum transfer and thus losses completely the effects of impingement. Since then in all the projects undertaken by the authors the impinging velocity, $u_{0}$, is employed as the criterion for design and scaling-up. This may be a typical instance showing that the scale-up method based on the $\pi$ law is invalid not only for most of chemical reaction systems but also for some of physical ones, like IS.

Mahajan and Kirwan [16] studied micromixing in the two jets mixer, as a part of fundamental work for preparation of ultrafine medicines. The mixer is actually a two-tangential impinging stream reactor without circulation. They obtained the data less than 200 milliseconds for the micromixing time under moderate conditions, and the data also about obey the relationship of $t_{M} \propto u_{0}^{-1.5}$.

The results of both the investigations mentioned above indicate clearly that LIS promotes micro-mixing very efficiently. Unfortunately, a new model for micromixing, correct and universal, cannot be established yet, and so further studies are needed.

3.3. Strong Pressure Fluctuation. Also, the interaction between opposing streams in LIS results in vibration of fluid elements and/or molecules, and the global behavior is just pressure fluctuation. Zhang et al. [17] and Sun et al. [18] studied pressure fluctuation in the same SCISR with accurate pressure probes of $1.6 \mathrm{~mm}$ in diameter. The major conclusions Sun et al. [18] obtained are the following

(1) There exists considerably strong pressure fluctuation in SCISR. The standard deviation of instantaneous pressure can achieve 200 to $300 \mathrm{~Pa}$, implying the maximum amplitude of fluctuation being about $1.6 \mathrm{kPa}$, under the condition of impinging velocity being about $0.2 \mathrm{~m} \cdot \mathrm{s}^{-1}$ only.

(2) The major frequency of fluctuation is concentrated in the range of $<1000 \mathrm{~Hz}$, while there are some lower peeks in acoustic wave range.
(3) The impinging velocity, $u_{0}$, exhibits significant positive effects on intensity and major frequency range of the fluctuation: both of them increase about linearly as $u_{0}$ increases, implying that $u_{0}$ is a very important operating parameter.

3.4. Promoting Process Kinetics. It is clear that both efficient micromixing and strong pressure fluctuation are resulted from the interaction between the two streams in impingement, including collision, pressing, and shearing between fluid elements, as mentioned above. Well micromixing must increase the possibility of collision between molecules. While pressure fluctuation, as the global behavior of molecules and/or fluid elements vibration, means a part of flowing energy converted into vibration energy of molecules and/or fluid elements. During the conversion, molecule energies and their profile may be changed so that more molecules achieve higher energy level. It is therefore inferred that the combined effect of strong micromixing and pressure fluctuation may increase the probability of effective collision and thus favors kinetics. This inference has been confirmed by experiments.

Wu et al. [19] and Zhou et al. [20] worked comparative studies on the kinetics of $\mathrm{Na}_{2} \mathrm{HPO}_{4}$ crystal growth and alcohol ester saponification.

The kinetics data of $\mathrm{Na}_{2} \mathrm{HPO}_{4}$ crystal growth were measured in the same SCISR operated at the impinging velocity ranged from 0.1 to $0.2 \mathrm{~m} \cdot \mathrm{s}^{-1}$ and a fluidized-bed crystallizer (FBC), respectively; while the latter can be considered as a classical device for the study of crystal growth kinetics. The values for the overall crystal-growth rate coefficient of hydrated $\mathrm{Na}_{2} \mathrm{HPO}_{4}$ measured in SCISR, $K_{\mathrm{IS}}$, at different temperatures in the range of 32.7 to $39^{\circ} \mathrm{C}$ are ranged from 6.61 to $25.32 \times 10^{-6} \mathrm{~m} \cdot \mathrm{s}^{-1}$, which are higher systematically by 15 to $20 \%$ than those in $\mathrm{FBC}, K_{\mathrm{FB}}$, under the same conditions; no obvious difference is found between the observed active energies measured in the two kinds of crystallizers.

The kinetics data of alcohol ester saponification were measured, respectively, in the SCISR with an effective volume 
of $3.6 \mathrm{~L}$ and a traditional stirred tank reactor (STR) of $0.6 \mathrm{~L}$ operated under the same conditions. The results indicate that the values for the rate constant of the secondorder irreversible reaction measured in SCISR, $k_{\mathrm{IS}}$, in the temperature range of 25 to $45^{\circ} \mathrm{C}$ are ranged from 0.175 to $0.758 \mathrm{~m}^{3} \cdot \mathrm{kmol}^{-1} \cdot \mathrm{s}^{-1}$, higher systematically than those in STR, $k_{\text {STR }}$, by over $20 \%$. For example, the value for $k_{\mathrm{IS}}$ at $25^{\circ} \mathrm{C}$ averaged over 9 sets of data is 0.21 ; while that for $k_{\mathrm{STR}}$ is 0.15 . And, again, no obvious difference is found between the active energies measured in the two reactors.

Later, a comparative investigation was also made for hydrolysis kinetics of sugar by Chen et al. and similar results were obtained, as reported in [21].

What can be concluded or, at least, inferred, from the results above are the following

(1) LIS promotes process kinetics significantly; or, more generally, process kinetics depends not only on the natures of the system involved and operating conditions such as temperature, and so forth, but also on flow configuration in the reactor employed.

(2) The fact that no obvious differences between the values for the active energies measured in different reactors or crystallizers are observed suggests that LIS increases the frequency factors, $K_{\mathrm{IS}}^{0}$ and $k_{\mathrm{IS}}^{0}$, but does not change the active energies. These phenomena support the inference that LIS increases effective collision probability in kinetic processes. $\mathrm{Wu}[3,5]$ discussed the reasons theoretically and briefly.

Also, the problem rises from the results: How to get, or what reactor should be used for, accurate kinetic data. SCISR cannot yet be considered as the ideal one for, at least, its micromixing time, $t_{M}$ is not zero under the operating conditions employed. It is somewhat unfortunate that SCISR could not be operated at higher impinging velocity for too much turbulence destroying stable operation. So, it is not quite sure whether the rate constants could be increased further as the impinging velocity increasing. SCISR has also other disadvantages for industrial application. To cover the problem of lower operable impinging velocity and the others, Wu and Zhou [22] developed the Vertical Circulative Impinging Stream Reactor (VCISR); Y. Wu and $\mathrm{X} . \mathrm{X} . \mathrm{Wu}$ [23] improved further the design and patented the Non rotating Flow Vertical Circulative Impinging Stream Reactor (NRFVCISR), which can be operated at much higher impinging velocity, and is to be used for kinetics studies. Its design and other applications will be discussed later.

Obviously, the features of IS, including GIS and LIS, described above have great potential of application, provided that the target systems are chosen properly. In fact, in a number of application cases they exhibited very well performances, as briefly described in what follows.

\section{Application of GIS: Wet FGD}

As mentioned in [4], the earliest industrial application of GIS is for combustion of powdery coal in Koppers-Totaek coalgasifier $[24,25]$ and somewhat later for grinding of solids [10].
In addition to the above mentioned applications, a remarkable application of GIS should be for flue gas desulfurization (FGD), a very important problem of environment protection as well known. Wu et al. [9] and Li. [27] investigated wet FGD by absorption in GIS, and excellent results were obtained.

4.1. Adaptability of GIS for FGD. As described above, GIS alone is suitable only for rapid or instant processes governed by gas-film diffusion.

With dilute $\mathrm{Ca}(\mathrm{OH})_{2}$-in-water suspension as the absorbent for wet FGD, the major reactions in liquid are instant and irreversible with new solid products forming $[9,28]$, and thus the process is controlled by diffusion through gas film. Therefore GIS is especially applicable for the mentioned goal.

4.2. Absorption Equipment for Pilot Plant Test. The equipment used for the pilot plant test of wet FGD is the patented impinging stream gas-liquid reactor (ISGLR) [29], as shown in Figure 3. It employs the basic flow configuration of horizontal coaxial two-impinging streams and has the important feature of few inner parts, resulting in reduced pressure drop, eased caking on the walls, and easier cleaning of the device. For atomization of the absorbent the patented eddy pressure nozzles [30] shown in Figure 4 are employed, which have simple structure and high energy efficiency. The main dimensions of the reactor processing about $500 \mathrm{~m} \cdot \mathrm{h}^{-1}$ of flue gas are: Diameter of absorption chamber $D_{a}=700 \mathrm{~mm}$, Height of cylinder vessel $H_{a}=950 \mathrm{~mm}$, Diameter of gas conduits $d_{0}=80 \mathrm{~mm}$, Height of gas conduit axis from the lower edge of the cylinder $h=400 \mathrm{~mm}$, and Impinging distance $S$ is adjustable from $4 d_{0}$ to $6 d_{0}$. For the details of the system scheme and experimental procedure one may refer to [9].

\subsection{Major Results of the Pilot Plant Test}

4.3.1. Overall Performance of the Reactor. The essential goal of the study is to develop FGD equipment of industrial interest, and so understanding its general performance is of significance. With fully mixed $\mathrm{SO}_{2}$ and air as the pseudoflue gas (concentration of $\mathrm{CO}_{2}$ equals zero), several sets of typical operation data measured in stable operations are listed in Table 1, which illustrate that the designed equipment has satisfactory overall performance and meets the requisitions well for wet FGD. Under moderate operating conditions, the content of $\mathrm{SO}_{2}$ in the cleaned gas can achieve a level lower than that permitted, and even at the mole ratio of $\mathrm{Ca} / \mathrm{S}$ as low as 1.0 , a sulfur-removal efficiency of near $90 \%$ can be obtained (see the data in the fourth row of Table 1). In addition, the pressure drop across the reactor is about $400 \mathrm{~Pa}$ only. This is a very important advantage for industrial application.

4.3.2. Mass Transfer Coefficients. As the most important indexes, both gas-film and volumetric mass transfer coefficients and the influences of some factors on them were investigated. The major results obtained are: 


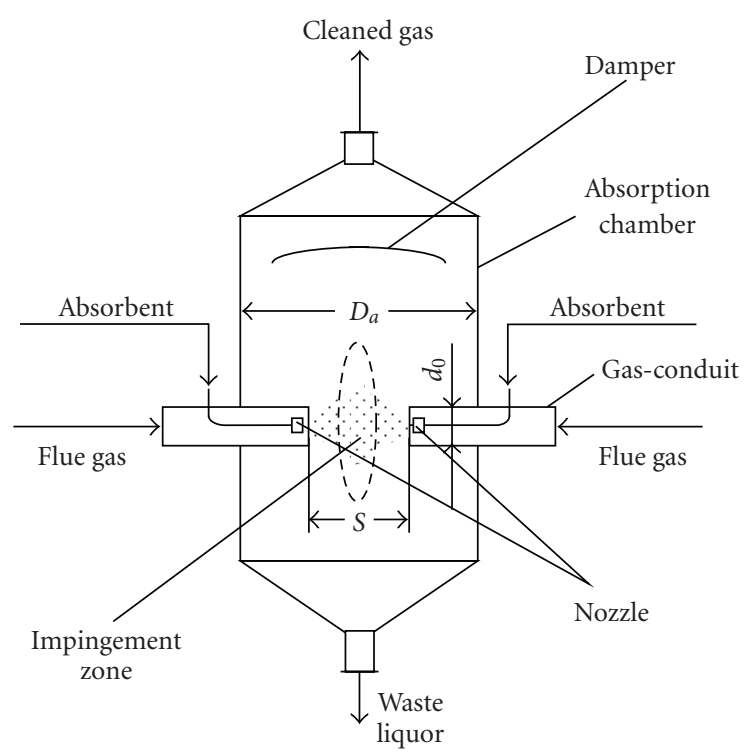

Figure 3: A brief view of GIS gas-liquid reactor.

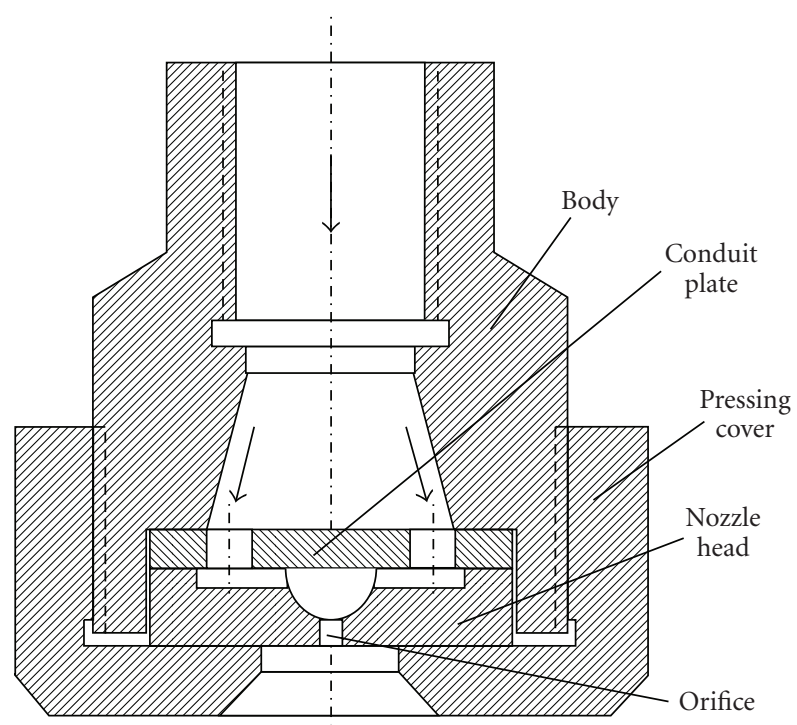

FIGURE 4: The eddy pressure nozzle.

TABLE 1: Typical operation data of the impinging stream FGD system.

\begin{tabular}{|c|c|c|c|c|c|c|c|c|}
\hline $\begin{array}{l}\text { Gas flow } \\
\text { rate } \\
\mathrm{m}^{3} \cdot \mathrm{s}^{-1}\end{array}$ & $\begin{array}{l}\text { Initial } \\
\mathrm{SO}_{2} \text { in } \\
\text { gas mg } \\
\mathrm{m}^{-3}\end{array}$ & $\begin{array}{l}\text { Flow rate ratio } \\
V_{L} / V_{G} \mathrm{~m}^{3} \cdot \mathrm{m}^{-3}\end{array}$ & $\begin{array}{l}\text { Mole ratio of } \\
\mathrm{Ca} / \mathrm{S} \mathrm{Mol} \cdot \mathrm{mol}^{-1}\end{array}$ & $\begin{array}{l}\text { Impinging } \\
\text { velocity } \\
\mathrm{m} \cdot \mathrm{s}^{-1}\end{array}$ & $\begin{array}{l}\text { Contacting } \\
\text { time, } s\end{array}$ & $\begin{array}{l}\text { Final } \mathrm{SO}_{2} \text { in } \\
\text { gas } \mathrm{mg} \cdot \mathrm{m}^{-3}\end{array}$ & $\begin{array}{l}\text { Efficiency } \\
\text { of sulfur- } \\
\text { removal, } \\
\%\end{array}$ & $\begin{array}{l}\text { Pressure } \\
\text { drop over } \\
\text { reactor, } \mathrm{Pa}\end{array}$ \\
\hline 0.08 & 3200 & 0.84 & 1.4 & 7.0 & 3.02 & 240 & 92.5 & 405 \\
\hline 0.08 & 2400 & 0.84 & 1.4 & 7.0 & 3.02 & 132 & 94.5 & 405 \\
\hline 0.08 & 2000 & 1.20 & 1.4 & 7.0 & 3.02 & 80 & 96.0 & 405 \\
\hline 0.08 & 2400 & 0.85 & 1.0 & 3.02 & 3.02 & 276 & 88.5 & 405 \\
\hline 0.10 & 2000 & 0.65 & 1.4 & 10.2 & 2.42 & 91.8 & 91.8 & 380 \\
\hline
\end{tabular}


TABLE 2: Comparison between volumetric mass transfer coefficients measured in ISGLR and RPB, respectively.

\begin{tabular}{|c|c|c|c|c|}
\hline Device & System & Rotary speed, rpm & $k_{L} a$ or $k_{G} a, \mathrm{~s}^{-1}$ & Reference \\
\hline ISGLR & $\mathrm{SO}_{2}-\mathrm{Ca}(\mathrm{OH})_{2}$ & 0 & $0.58-1.04$ & {$[9]$} \\
\hline RPB & $\mathrm{CO}_{2}-\mathrm{NaOH}$ & $\sim 1400$ & $0.21-0.44$ & {$[31]$} \\
\hline $\mathrm{RPB}$ & $\mathrm{CO}_{2}-\mathrm{NaOH}$ & 1400 & 0.35 & {$[32]$} \\
\hline $\mathrm{RPB}$ & $\mathrm{CO}_{2}-\mathrm{NaOH}$ & 1550 & 1.14 & {$[26,33]$} \\
\hline $\mathrm{RPB}$ & $\mathrm{CO}_{2}-\mathrm{NaOH}$ & 1369 & 0.66 & [34] \\
\hline
\end{tabular}

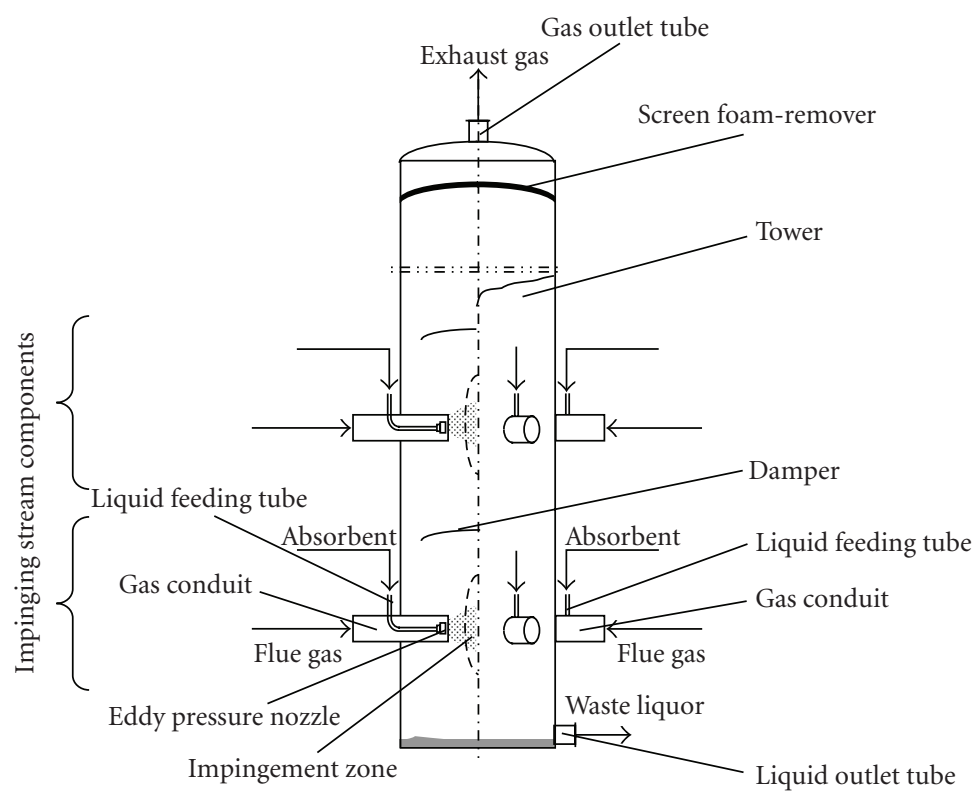

FIgURE 5: Brief view of HFISGLR 1-tower; 2-screen foam-remover; 3-gas outlet tube; 4-liquid outlet tube; 5-gas conduit; 6-eddy pressure nozzle; 7-liquid feeding tube; 8-damper I, II. ... impinging stream components.

(1) The impinging velocity, $u_{0}$, has strong influence on both gas-film and volumetric mass transfer coefficients, $k_{G}$ and $k_{G} a$. The relationship between $k_{G}$ and $u_{0}$ is fitted very well by

$$
k_{G}=2.9 \times 10^{-4} u_{0}^{1.75821}
$$

with a standard deviation of SD $=2.45 \times 10^{-4} \mathrm{~m} \cdot \mathrm{s}^{-1}$. The power 1.75821 indicates $u_{0}$ that is a very strong effecting factor and, consequentially, should be an important operating parameter.

(2) With the impinging velocity $u_{0}$ ranged from 5.53 to $16.62 \mathrm{~m} \cdot \mathrm{s}^{-1}$, the volumetric mass transfer coefficient, $k_{G} a$, measured is in the range of 0.577 to $1.037 \mathrm{~s}^{-1}$, while the gas-film one, $k_{G}$, is ranged from 0.00641 to $0.0416 \mathrm{~m} \cdot \mathrm{s}^{-1}$, showing again clearly that GIS enhances mass transfer very efficiently.

(3) Essentially, $\mathrm{SO}_{2}$ concentration in flue gas has no influence on $k_{G}$, suggesting that the process does be controlled by diffusion through gas film.

Table 2 gives a comparison between volumetric mass transfer coefficients measured in ISGLR and the rotary packed bed (RPB), respectively; while the latter is another kind of device enhancing transfer also very efficiently and receives increasing attention in the recent years. It is obvious from the table that the two-kind equipments exhibit essentially the same ability in enhancing transfer between gas and liquid phases. On the other hand, in operation, ISGLR itself is motionless, while RPD is rotating at considerably high speed and, consequentially, needs higher capital cost, maintains fee, and consumes more energy.

4.4. Development of GIS Device for Wet FGD of Full Scale. The device shown in Figure 3 cannot be an "industrial" one for wet FGD, because its capacity of gas flowrate is limited. As well known, huge amount of flue gas is exhausted every hour from each power station of coal burning. To solve this problem, Wu et al. [35] designed and patented the Huge Flowrate Impinging Stream Gas-Liquid Reactor (HFISGLR), as shown in Figure 5. It employs the flow configuration of multigroup one over one with each group consisting of multipair of horizontal coaxial two impinging streams so that each pair of impinging streams is operated co currently and works on the same principles and in same mode. Technically, both the methods of scale-up and number-up are used in the design and so, to a great extent, the so-called "scaling-up effect" is avoided. 


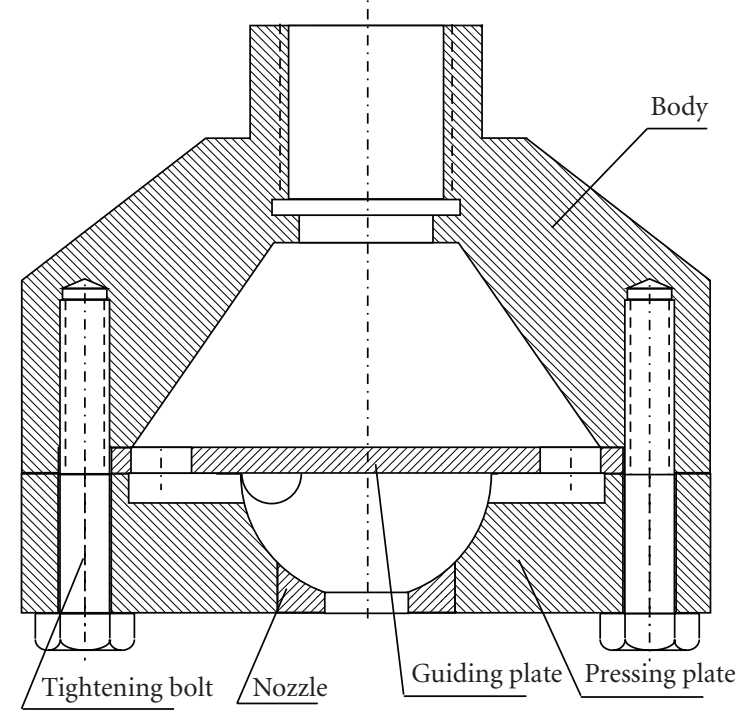

FIgURE 6: The large flowrate eddy pressure nozzle [26].

Atomization of absorbent is an important operation in the wet FGD technology discussed here. GIS itself calls finely dispersed absorbent, on one hand; while atomization provides great interface area to increase transfer rate, on the other. Therefore atomizer is an important auxiliary device to HFISGLR. Since flue gas from coal-burning is generated in huge amount, the amount of absorbent needing to be atomized is also very large, even if a small liquid-to-gas ratio is used. So atomizers of high production must be used. Otherwise, over thousand atomizers may be needed in one GIS absorber, making installation and maintenance extremely difficult. Based on plentiful experience obtained in successful industrial applications of the eddy pressure nozzle developed earlier [30], the large flowrate eddy pressure nozzle was developed and patented by Y. Wu and X. H. Wu [36], as shown in Figure 6, which keeps the advantages of very short passageway at high pressure and thus high energy efficiency, and can atomize up to $20 \mathrm{~m}^{3}$ of absorbent per hour.

The combination of HFISGLR with the large flowrate eddy pressure nozzles forms a novel technology for wet FGD. In comparison with the most popular technology for wet FGD, at least in China, the so-called limestone-gypsum (LSG) process, in which fine limestone powder-in-water suspension is used as the absorbent and absorption is carried out in a spray tower, the novel technology is predicted to have the following major outstanding advantages: (1) Very low liquid-to-gas ratio and so great power saving; (2) Easy separation of reaction product and reduced jam possibility of equipment; (3) high volumetric efficiency and reduced absorber volume. The first set of wet FGD system of quasiindustrial scale employing the novel technology described here is being designed and to be built in the near future.

\section{Development and Applications of LIS Devices}

5.1. Application of SCISR in Preparation of Ultrafine Powders. SCISR shown in Figure 2 is the earliest design of LIS device.
In addition to the fundamental studies mentioned above, it was used for preparations of a number of ultrafine materials by reaction precipitation, and very well results were obtained.

5.1.1. Adaptability of LIS for the Goal. Reaction precipitation is an important category among various methods for preparation of ultrafine materials because of its economic reasonability and convenient and feasible operation. Essentially, the major process in this method is crystallization from a solution. For production of ultrafine product with narrow size distribution the key is to create high and uniform supersaturation environment for precipitation. In addition, the surface of nucleus or microparticles newly formed is very active and so usually needs to be aged for certain time to avoid or ease assembling of new microcrystals with each other to form larger particles.

High (average) supersaturation can always be achieved without difficulty; while uniform one is not easy. As mentioned above, SCISR, one of LIS devices, has the feature of very efficient micromixing and thus can provide the condition of very uniform supersaturation. On the other hand, SCISR has the special flow configuration of circulative perfect mixing-plug flow (essentially no mixing) in series [38]. To an extent the flow through the region without mixing with extremely low supersaturation after nucleation favors aging of microparticles newly formed. Therefore SCISR is especially suitable for preparation of ultrafine powders by reaction precipitation, and this has been verified by a number of preparation investigations, as partly described below.

5.1.2. "Ultrafine" White Carbon Black. With SCISR with an effective volume of 3.6 L shown in Figure 2, Wu and Chen [39] prepared "ultrafine" white carbon black by the common (one step) precipitation process, and obtained the following major results. (1) In batch operations, the products consist of assembled particles sized from 0.5 to 2.0 with the averages of 


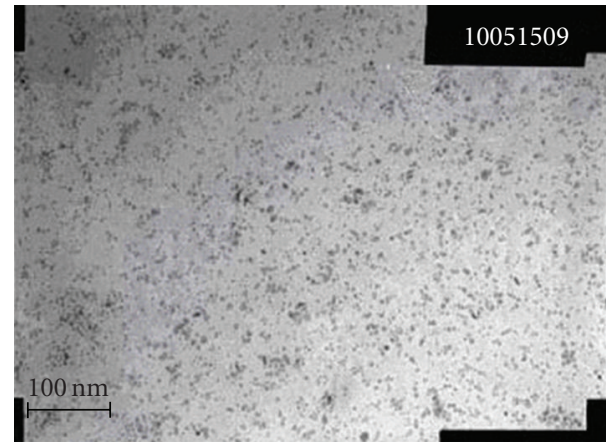

(a) Sphere form

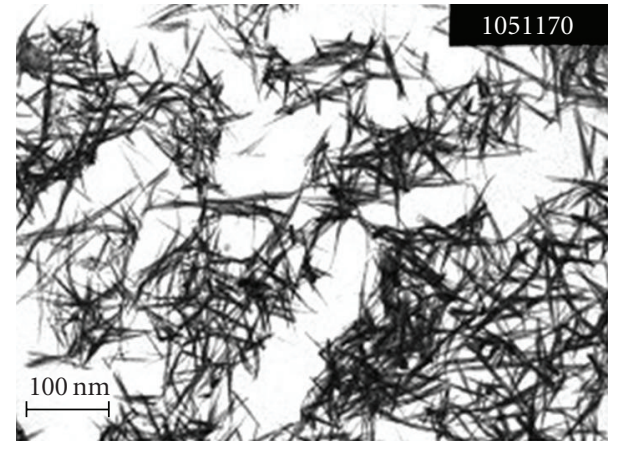

(b) Needle form

FIGURE 7: TEM photos of products with different forms.

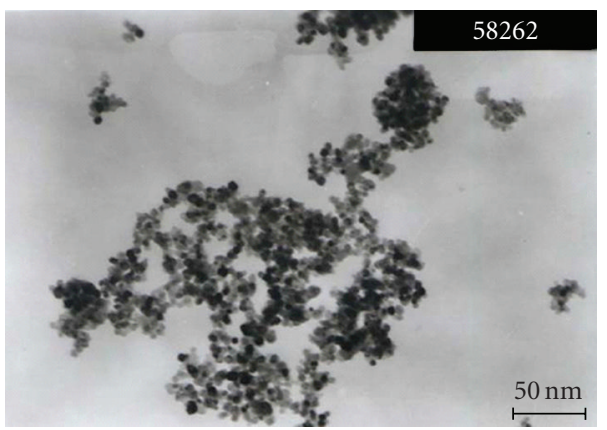

(a) Calcined at $600^{\circ} \mathrm{C}$

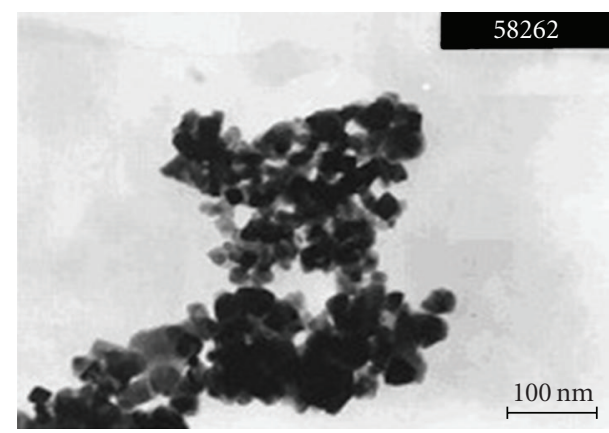

(b) Calcined at $800^{\circ} \mathrm{C}$

Figure 8: TEM photos of Nano Titania calcined at 600 and $800^{\circ} \mathrm{C}$, respectively.

1.1 to $1.6 \mu \mathrm{m}$, and the reproducibility and regularity of data are very well. (2) Continuous operation of SCISR yields the same results. (3) The sizes of the reaction products are stable and do not change during the treatment after reaction, for example, filtering and drying. It should be mentioned that the particles in the products produced by the same process with the traditional stirred tank reactor (STR) are averagesized 30 to $50 \mu \mathrm{m}$ with much wider size distribution.

\subsubsection{Nanocopper and Copper-Silver Double Metal Powders.} Chen [40] prepared Nanocopper powders by $\mathrm{CuCl}_{2}$ reduction precipitation in the same SCISR with $\mathrm{KBH}_{4}$ as the reducing agent. The $\mathrm{KBH}_{4}$ solution at certain $\mathrm{pH}$ adjusted with $\mathrm{KOH}$ is transferred into SCISR; drop $\mathrm{CuCl}_{2}$ solution into the reaction mixture at a controlled rate to carry out the reaction at constant temperature for certain time; then separate, wash, and dry the precipitate to yield product, which is then detected with TEM and XRD for size and appearance. The major results Chen obtained are as follows.

(1) The product obtained under the optimized conditions is average sized $5.1 \mathrm{~nm}$, while the average size of the mixed products from over 20 repeated runs is less than $10 \mathrm{~nm}$, suggesting well stability of operation. A comparison of the mean size of the products with those by other processes and/or reactors is given in Table 3.

(2) Using the mixed product in lubricating grease as an additive leads to an increase in lubricating efficiency by $50 \%$, higher than those with grinded Nano copper powder as the additive by about $20 \%$ [47].

(3) By properly controlling the conditions both the products of sphere and needle forms can be obtained, as shown in Figure 7.

Chen also studied the preparation of $\mathrm{Cu}-\mathrm{Ag}$ double metal powder from Nano copper particles of sphere form by substitution, with Ag-ammonium solution as the reagent and gelatin as the protecting agent, in order to improve anti oxidation nature of product. A comparison of the experimental product with those by other methods and/or equipments is given in Table 4. The data show that the product from SCISR is the finest with narrowest size distribution; while its operation conditions are very moderate, for example, the impinging velocity is about $0.2 \mathrm{~m} \cdot \mathrm{s}^{-1}$ only.

5.1.4. Nano-Titania. Li [51] studied the preparation of Nano Titania by $\mathrm{TiCl}_{4}$ hydrolyzation precipitation in the same SCISR. Through a widely searching procedure, the major optimal conditions are determined to be $\mathrm{pH}=$ 4.0 , reaction temperature $=84^{\circ} \mathrm{C}$, initial concentration of $\mathrm{TiCl}_{4}=1.4 \mathrm{kmol} \cdot \mathrm{m}^{-3}$, neutralization time $=400 \mathrm{~s}$, reaction time after neutralization $=1$ hour, and impinging velocity is about $0.28 \mathrm{~m} \cdot \mathrm{s}^{-1}$. 
TABLE 3: Comparison of average sizes of nanocopper products obtained by various processes and with various reactors.

\begin{tabular}{lccccc}
\hline Copper salt & Reducing agent & Temperature, ${ }^{\circ} \mathrm{C}$ & Reactor & Mean size of product, $\mathrm{nm}$ & Reference \\
\hline $\mathrm{CuSO}_{4}$ & $\mathrm{HCHO}$ & 70 & STR & 100 & {$[41]$} \\
$\mathrm{CuSO}_{4}$ & Ascorbic acid & 85 & STR & $\geq 500$ & {$[42]$} \\
$\mathrm{CuSO}_{4}$ & $\mathrm{~N}_{2} \mathrm{H}_{4} \cdot \mathrm{H}_{2} \mathrm{O}$ & 60 & STR & $50-500$ & {$[43]$} \\
$\mathrm{CuSO}_{4} \cdot 5 \mathrm{H}_{2} \mathrm{O}$ & $\mathrm{NaH}_{2} \mathrm{PO}_{2}$ & $55-65$ & STR & 50 & {$[44]$} \\
$\mathrm{CuSO}_{4}$ & $\mathrm{KBH}_{4}$ & 20 & STR & $40-100$ & {$[45]$} \\
$\mathrm{CuCl}_{2}$ & $\mathrm{KBH}_{4}$ & 20 & SCISR & $\sim 10$ & {$[40]$} \\
$\mathrm{CuCl}_{2}$ & $\mathrm{Na}_{2} \mathrm{~S}_{2} \mathrm{O}_{4}$ & $60-70$ & STR & 20 & {$[46]$} \\
\hline
\end{tabular}

TABLE 4: Comparison between Cu-Ag double metal powders obtained by various processes and/or with various reactors.

\begin{tabular}{|c|c|c|c|c|c|c|}
\hline \multicolumn{2}{|c|}{$\begin{array}{l}\text { Reducing agent and/or reactor } \\
\text { for preparation of nano copper }\end{array}$} & $\begin{array}{l}\text { Average size of } \\
\text { nano copper }\end{array}$ & $\begin{array}{l}\text { Amount of Ag } \\
\text { coated, \%mass }\end{array}$ & $\begin{array}{l}\text { Mean size of } \\
\text { Cu-Ag powder }\end{array}$ & $\begin{array}{c}\text { Antioxidation } \\
\text { nature at room } \\
\text { temperature }\end{array}$ & Reference \\
\hline \multicolumn{2}{|c|}{$\mathrm{NaH}_{2} \mathrm{PO}_{2}$} & $\sim 50 \mathrm{~nm}$ & $22-85$ & $\geq 100 \mathrm{~nm}$ & Yes & {$[48]$} \\
\hline \multicolumn{2}{|c|}{$\mathrm{N}_{2} \mathrm{H}_{4} \cdot \mathrm{H}_{2} \mathrm{O}$} & $50-500 \mathrm{~nm}$ & - & $\geq 300 \mathrm{~nm}$ & Yes & [49] \\
\hline \multicolumn{2}{|c|}{ Ascorbic acid } & $\geq 500 \mathrm{~nm}$ & $\sim 38.84$ & $\geq 500 \mathrm{~nm}$ & Yes & [42] \\
\hline \multirow{2}{*}{$\mathrm{KBH}_{4}$} & STR & $\sim 45 \mu \mathrm{m}$ & $\sim 15.22$ & $\sim 45 \mu \mathrm{m}$ & Yes & {$[50]$} \\
\hline & SCISR & $\sim 10 \mathrm{~nm}$ & $\sim 30$ & $5-30 \mathrm{~nm}$ & Yes & [40] \\
\hline
\end{tabular}

The sizes and crystalline types of products obtained under the optimal conditions while calcined at different temperatures are listed in Table 5; the yield of Ti is 0.962 . From the results reported up to date, the products by Li are the finest. The TEM photos of products calcined at 600 and $800^{\circ} \mathrm{C}$, respectively, are shown in Figure 8.

To verify the superiority of the reactor, a comparative study was also made in the SCISR with an effective volume of $3.6 \mathrm{~L}$ and a traditional stirred tank reactor (STR) of 0.6 $\mathrm{L}$, respectively, under the same conditions. The results are shown in Table 6. Obviously, SCISR is much prior to STR in both Ti-yield and particle sizes of product.

5.1.5. Nano-Hydroxyapatite. Hydroxyapatite (HAP), $\mathrm{Ca}_{5}$ $\left(\mathrm{PO}_{4}\right)_{3}(\mathrm{OH})$ or $\mathrm{Ca}_{10}\left(\mathrm{PO}_{4}\right)_{6}(\mathrm{OH})_{2}$, has important application in medicine because of its well bio compatibility. Yuan et al. [52] studied its synthesis in the same SCISR by the double decomposition between $\left(\mathrm{NH}_{4}\right)_{2} \mathrm{HPO}_{4}$ and $\mathrm{Ca}\left(\mathrm{NO}_{3}\right)_{2}$ precipitation. The procedure is as follows. Put $\mathrm{Ca}\left(\mathrm{NO}_{3}\right)_{2}$ solution with the concentration of $0.28 \mathrm{~mol} \cdot \mathrm{L}^{-1}$ into SCISR. Under the condition of circulative flow driven by the propellers and at room temperature, drop $\left(\mathrm{NH}_{4}\right)_{2} \mathrm{HPO}_{4}$ solution of concentration $1.07 \mathrm{~mol} \cdot \mathrm{L}^{-1}$, essentially in stoichiometric amount, into the reactor. Adjust $\mathrm{pH}$ of the mixture with aqua ammonia. Continue the reaction for 10 minutes. Transfer the reacted mixture into a three-neck flask with agitator to heat it for a certain time under the condition of back-flow. Age, fitter, wash, and dry to yield the final product.
The influences of mixture $\mathrm{pH}$, dropping rate of $\left(\mathrm{NH}_{4}\right)_{2} \mathrm{HPO}_{4}$ solution, and heating time after reaction are examined and the products are characterized by energy spectrum, thermal difference, infrared spectroscopy with Fourier transformation, X-ray diffraction, scanning, and transmission electron microscopy to determine composition, mainly $\mathrm{Ca} / \mathrm{P}$ mole ratio, and appearance of the microcrystals. The optimal conditions determined in the primary study are Dropping rate of $\left(\mathrm{NH}_{4}\right)_{2} \mathrm{PO}_{4}$ solution $54 \mathrm{~mL} \cdot \mathrm{min}^{-1}, \mathrm{pH}$ of mixture 12.5, back-flow (heating) time 3-4 hours, and the amounts of $\mathrm{Ca}\left(\mathrm{NO}_{3}\right)_{2}$ and $\left(\mathrm{NH}_{4}\right)_{2} \mathrm{HPO}_{4}$ in stoichiometric ratio.

The TEM photo of the product prepared under primarily optimized conditions is shown in Figure 9. It can be seen that the product consists of crystals of about $15 \mathrm{~nm}$ in diameter and 50-60 $\mathrm{nm}$ long, with uniform sizes and regular appearance. The composition analysis results in the mole ratio of $\mathrm{Ca} / \mathrm{P}=1.683$, suggesting that the product contains a little excess calcium; while the results of X-ray diffraction analysis indicate that the product has complete Hydroxyapatite crystalline. These results show that the product prepared is ideal nano-Hydroxyapatite.

In addition, with the same SCISR Zhou et al. studied experimentally the preparation of nanomagnesium oxide [53], nanobarium titanate [54], nanozinc oxide [37], nanostrontium titanate [55], and so forth, and all the studies yield well results.

All the results related to the subject indicate that LIS is a powerful tool for preparation of ultrafine materials by reaction precipitation, and that improvement of technical 
TABle 5: Particle sizes and crystalline types of nano-Titania calcined at different temperatures.

\begin{tabular}{ccc}
\hline Temperature, ${ }^{\circ} \mathrm{C}$ & Mean size, $\mathrm{nm}$ & Crystalline type \\
\hline No calcined & Immeasurable & Amorphous \\
400 & 5.47 & Incomplete anatase \\
600 & 8.84 & Anatase \\
800 & 26.84 & Rutile \\
\hline
\end{tabular}

TABLE 6: Comparative results of SCISR with STR.

\begin{tabular}{lcccc}
\hline Reactor & Yield of Ti & Maximum & $\begin{array}{c}\text { Size of } \mathrm{TiO}_{2}, \mathrm{~nm} \\
\text { Minimum }\end{array}$ & Average \\
\hline SCISR & 0.962 & 16.0 & 2.0 & 5.68 \\
STR & 0.927 & 20.85 & 3.34 & 11.28 \\
\hline
\end{tabular}

device is also an effective way to enhance preparation technologies of Nano materials.

\subsection{Development and Applications of New LIS Reactor}

5.2.1. Improvement of LIS Reactor Design. As described above, SCISR has been used in a number of fundamental and applied investigations and has exhibited excellent performance in preparation of ultrafine powders. While it was also found to have some disadvantages caused by its horizontal structure, such as the following (1) The operating impinging velocity, $u_{0}$, is limited to about $0.2 \mathrm{~m} \cdot \mathrm{s}^{-1}$ only for larger $u_{0}$ leads to strong turbulent waves at liquor surface in the reactor and thus poor stability of operation; (2) the walls of the container include several plates so that it is somewhat difficult to construct the reactor to be operated under pressure; (3) because of being submerged in the reaction mixture, the bearings of propellers are easy to be scuffed in the case of the mixture containing solid particles, leading to troubles in maintenance, and even leakage. Multiple improvement was made to solve these problems and, as the final version, the nonrotating flow vertical circulative impinging stream reactor (NRFVCISR) was developed and patented [56], as shown in Figure 10.

The reactor employs the flow configuration of vertical co axial two impinging streams plus circulation after impingement. The basic design is as follows. The upper and lower drawing tubes of identical smaller diameter are placed co axially in a reaction container of larger diameter. Two propellers with opposing screw directions are mounted at the inlets of the tubes, respectively, on the same shaft and driven by the same motor. The streams are drawn by the propellers and transported through the drawing tubes towards the center of the container and impinge against each other to form vertical coaxial two impinging streams. After impingement, the streams flow through the annular chambers between the drawing tubes and the wall of the container to circulate. Thus, like SCISR, NRFVCISR has the special flow configuration of circulative complete mixing flow-no mixing flow in series. The circulation is originally arranged for increasing residence time to meet requirement of most reaction systems, while it is of especial significance for certain systems, as will be described later.

Because the impingement plane is at a depth far from the level, NRFVCISR can be operated at much higher impinging velocity stably; while the placement of bearing being over the reaction mixture keeps from possible detrition and leakage.

On the other hand, since the two propellers have opposing screw directions while are driven by the same shaft, their rotation will cause both axial flows in opposite directions and rotating flows in the same tangential direction. As the result of the superposition of the two rotary momentums, very strong rotating flow occurs, yielding great centrifugal force and thus concave level with large height difference, destroying normal operation. In the design of NRFVCISR, as the measure of restraining rotating flow, several vertical buffers are mounted on the inside walls of the drawing tubes. Because of acting at the beginning, they restrain the rotating flow very efficiently without additional energy consumption.

The application cases described below show that the design of NRFVCISR has attained completion.

5.2.2. Application of NRFVCISR in the Pilot Plant Test of "Ultrafine" White Carbon Black Preparation. According to the results of batch runs reported in [39], which are described briefly in Section 5.1.2, the authors of the present paper are requested by a company to carry out a pilot plant test on preparation of "ultrafine" white carbon black by the common (one step) reaction-precipitation process for data of full-scale system design. The major reaction involved is well known as

$$
m \mathrm{SiO}_{2} \cdot \mathrm{Na}_{2} \mathrm{O}+\mathrm{H}_{2} \mathrm{SO}_{4} \longrightarrow m \mathrm{SiO}_{2} \downarrow+\mathrm{Na}_{2} \mathrm{SO}_{4}+\mathrm{H}_{2} \mathrm{O} \text {. }
$$

Instead of SCISR, the pilot plant test employs the NRFVCISR with an effective volume of $0.3 \mathrm{~m}^{3}$ for the reaction, of which the production is 200-300 tones per year. The system scheme is briefly shown in Figure 11.

Near 30 runs were made for both experimental data and probationary product. The measured average sizes of products from a part of runs, which are for probationary product and reconfirming the feasibility and so employ essentially the same conditions, are listed in Table 7; while 


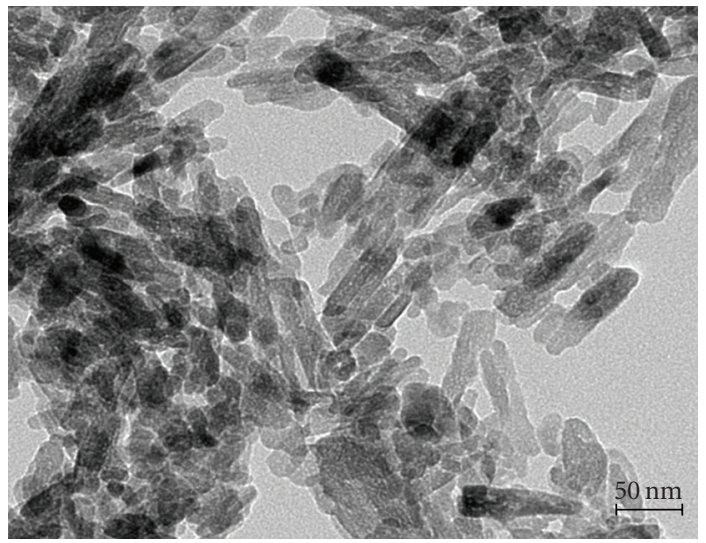

FIGURE 9: TEM photo of nano Hydroxyapatite prepared under primarily optimized conditions.

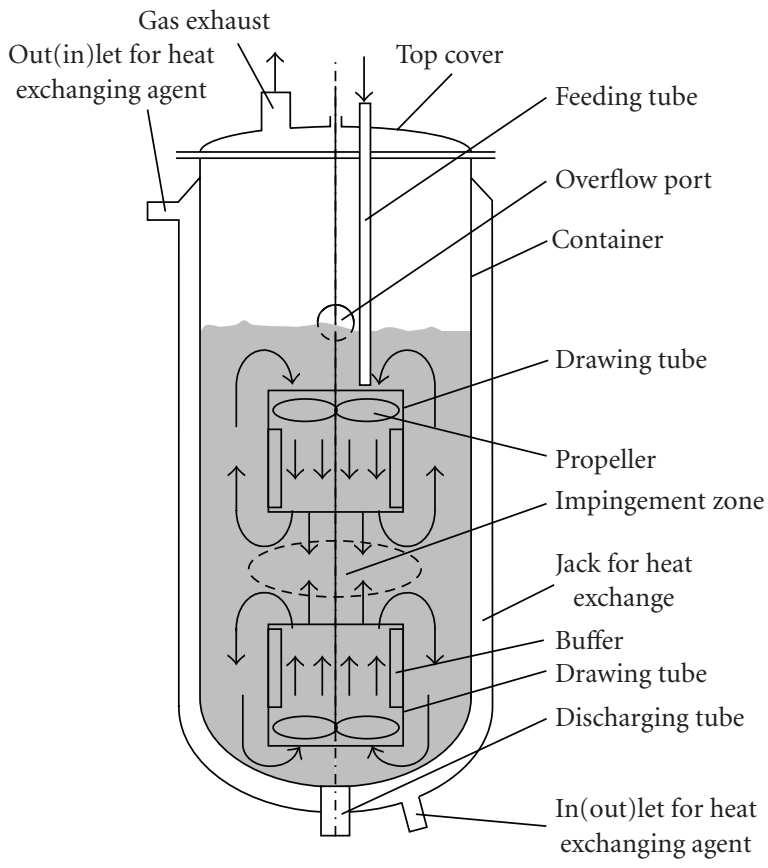

FigURE 10: The non-rotating flow vertical circulative impinging stream reactor [37].

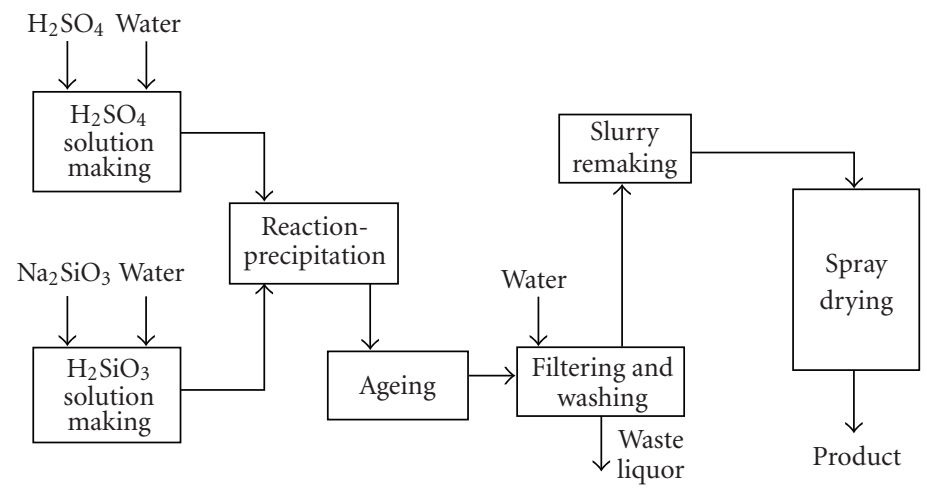

Figure 11: Principle system scheme of pilot plant test for preparation of "ultrafine" white carbon black by impinging stream reaction-precipitation. 


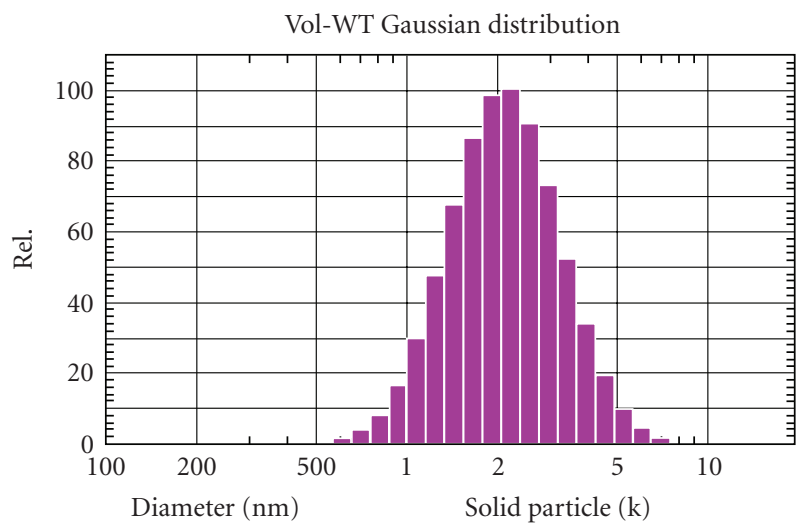

FIGURE 12: Size distribution probability of typical "ultrafine" white carbon black.

the size distribution of a typical product is given in Figure 12. The specific surface area of products is as high as 820 to $860 \mathrm{~m}^{2} \cdot \mathrm{g}^{-1}$.

From the data listed in Table 7 and those measured for the specific surface area it follows that, with the nonrotating flow vertical circulative impinging stream reactor as the reaction-precipitation device and simply by the common (one step) precipitation process, the pilot plant system yields constantly "ultrafine" white carbon black average sized 2 to $3 \mu \mathrm{m}$ with narrow size distribution (see Figure 12). As described above, by the same process, the traditional stirred tank reactor (STR) produces the products average-sized 30 to $50 \mu \mathrm{m}$ and are incomparable with those from NRFVCISR.

On a quasi commercial scale, the results of the pilot plant test above prove again that the superior micromixing property favors reaction-precipitation processes to produce ultrafine powders with smaller particle sizes and narrower size distribution.

\subsubsection{Application of NRFVCISR to Pentaerythritol Production} of Large Scale. The application of NRFVCISR in commercial production of pentaerythritol as the condensation reactor is a very successful example.

In 2008, a group company built a new production system for pentaerythritol with monopentaerythritol as the main product. Monopentaerythritol and bipentaerythritol are produced by condensation of acetaldehyde with formaldehyde and caustic soda. This is a complex system, and the main reactions are

$$
\begin{aligned}
& \mathrm{CH}_{3} \mathrm{CHO} \stackrel{\mathrm{HCHO}^{\circ} \mathrm{OH}^{-}}{\longrightarrow} \mathrm{CH}_{2} \mathrm{OH}-\underset{\text { (I) }}{\mathrm{CH}_{2}}-\mathrm{CHO} \stackrel{\mathrm{HCHO}^{-\mathrm{OH}^{=}}}{\longrightarrow}\left(\mathrm{CH}_{2} \mathrm{OH}\right)_{2} \mathrm{CH}-\mathrm{CHO}
\end{aligned}
$$

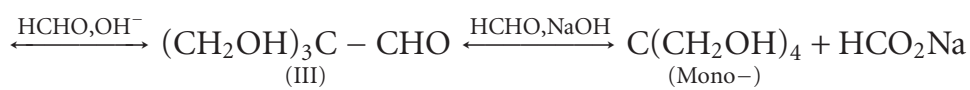

$$
\begin{aligned}
& \mathrm{CH}_{3} \mathrm{CHO} \stackrel{\mathrm{HCHO}, \mathrm{OH}^{-}}{\longrightarrow} \mathrm{CH}_{2} \mathrm{OH}-\mathrm{CH}_{2}-\mathrm{CHO} \stackrel{\mathrm{OH}^{-}}{\longrightarrow} \mathrm{CH}_{2}=\underset{(\mathrm{IV})}{\mathrm{CH}}-\mathrm{CHO}+\mathrm{H}_{2} \mathrm{O} \\
& (\mathrm{I})+(\mathrm{IV}) \stackrel{\mathrm{OH}^{-}}{\longrightarrow} \mathrm{O}\left(\mathrm{CH}_{2}-\mathrm{CH}_{2}-\mathrm{CHO}\right)_{4} \\
& \stackrel{6 \mathrm{HCO}, \mathrm{NaOH}}{\longrightarrow}\left(\mathrm{CH}_{2} \mathrm{OH}\right)_{3} \mathrm{CCH}_{2} \mathrm{OCH}_{2} \mathrm{C}\left(\mathrm{CH}_{2} \mathrm{OH}\right)_{3}+\mathrm{HCO}_{2} \mathrm{Na}
\end{aligned}
$$

The reactions are carried out with large excess of formaldehyde, and acetaldehyde is the key component. Other by-reactions are also possible to occur. If acetaldehyde at high concentration contacts caustic soda directly, undesired by-reactions will occur to produce colored by-products, resulting in reduced yield, purity and, whiteness of product with poor market competition ability. Therefore feeding to and mixing in the condensation reactor are of essential importance.
The company is the earliest one producing pentaerythritol in China, and its skills including achieved yield, and so forth, are in the head, at least, in China. The original production system employs special stirred tank reactors (STRs) with external circulation; multiagitator, each contains several paddles of different forms, is used in the tank in order to enhance mixing. The acetaldehyde is fed into recycled mixture, and so the feeding point is far from that for caustic soda. 
TABLE 7: Average sizes of products from a part of runs.

\begin{tabular}{lllllll}
\hline Run nos. & $\begin{array}{l}\text { Concentration } \\
\text { of } \mathrm{Na}_{2} \mathrm{SiO}_{3} \\
\mathrm{SiO}_{2} \% \mathrm{~m}\end{array}$ & $\begin{array}{l}\text { Operating conditions } \\
\text { of } \mathrm{H}_{2} \mathrm{SO}_{3} \% \mathrm{~m}\end{array}$ & $\begin{array}{l}\text { Reaction } \\
\text { temperature }\end{array}$ & $\begin{array}{l}\text { Time for adding } \\
\text { acid S }\end{array}$ & $\begin{array}{l}\text { Reaction time } \\
\text { min }\end{array}$ & $\begin{array}{l}\mu \mathrm{m} \\
\mu \text { size }\end{array}$ \\
\hline 14 & 35 & 55 & $8-11$ & $>20$ & 10 & 2.4885 \\
15 & 35 & 55 & $8-11$ & $>20$ & 10 & 2.2698 \\
16 & 35 & 55 & $7-10$ & $15-20$ & 20 & 2.3120 \\
17 & 35 & 55 & $7-10$ & $15-20$ & 20 & 2.9426 \\
18 & 35 & 55 & $7-10$ & $15-20$ & 20 & 2.0024 \\
19 & 35 & 55 & $7.1-10.5$ & $15-20$ & 20 & 2.4689 \\
20 & 35 & 55 & $7.1-10.5$ & $15-20$ & 20 & 1.7418 \\
21 & 35 & 55 & $7.1-10.5$ & $15-20$ & 20 & 2.2162 \\
\hline
\end{tabular}

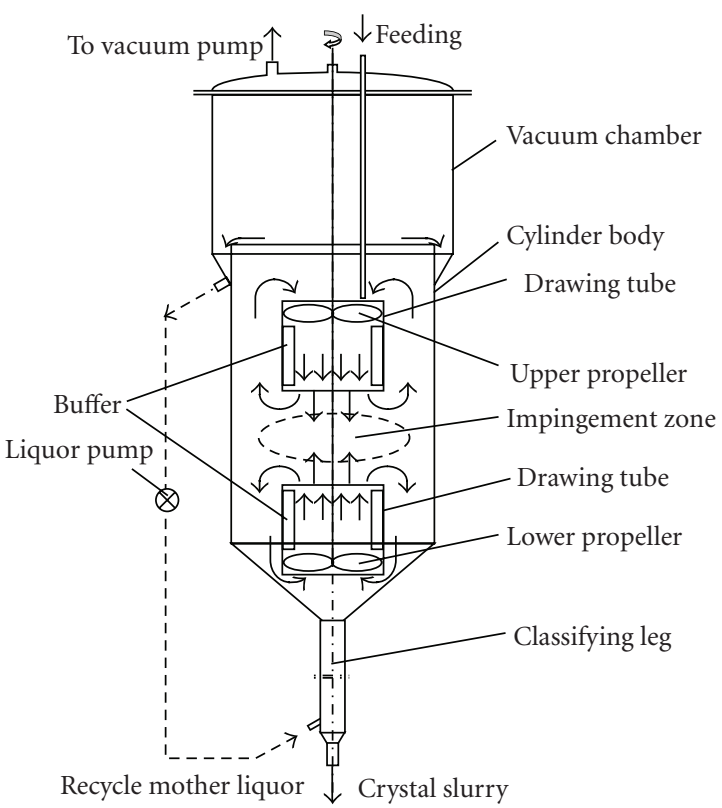

FIGURE 13: Non-rotating flow impinging stream crystallizer.

In the new system, three nonrotating vertical circulative impinging stream reactors (NRFVCISRs), effective volume of $28 \mathrm{~m}^{3}$ each, are employed for condensation. The acetaldehyde and caustic soda are fed at the inlets of the upper and lower drawing tubes, respectively; a coil cooler is settled between the wall of the container and the drawing tubes to remove the great reaction heat.

Up to date the reactors have been operated constantly for over one year without any fault, and exhibited excellent performances greatly prior to the stirred tank reactor with external circulation in the following.

(1) By-Reaction(s) Being Effectively Restrained. This is expressed by the following. (a) The chromaticity of product is obviously reduced, and the whiteness of the products primarily crystallized and recrystallized is higher by 35 and 25 (Pt-Co standard color), respectively, than those from the original production system, and so the appearance of product is significantly improved. (b) The consumption of acetaldehyde is reduced to an extent, and the statistic yield is increased by $1.1 \%$.

(2) Great Power Saving. The installed total capacity for the STR with external circulation in the old system is $70 \mathrm{~kW}$; while that designed for NRVCISR is $10 \mathrm{~kW}$ only for the same production, and the practically installed as requested by the user is $15 \mathrm{~kW}$.

The operation for long term shows that the feeding and mixing performances of NRFVCISR are very well. It is evaluated that, at an impinging velocity of $0.3 \mathrm{~m} \cdot \mathrm{s}^{-1}$, the internal recycling ratio can be as high as about 100; while the external one for the STR is designed as 20 , and can never be so high as the former.

NRFVCISR provides the superior features of efficient micromixing and strong pressure fluctuation, the inherent features of LIS, and so large recycling ratio although; its 
power consumption is very small. This is because of the special mode of fluid transportation. As well known, the propellers transport the reaction mixture at very small head that is needed to cover the resistance only. Generally, NRFVCISR needs an installed total capacity under one third of that for STR of the same effective volume without external circulation, and so its energy-saving effect is significant.

Because of its high efficiency, great energy saving, and the special functions in producing finer nanomaterials and enhancing selectivity of reactions, and so forth, NRVCISR can be expected to be the ideal substitute of the traditional stirred tank reactor (STR).

5.3. Development and Applications of LIS Crystallizer. Crystallization is an important unit operation for producing number solid products. The major aim of the operation is to obtain product with larger and uniform particle sizes; while the key is to create proper and uniform supersaturation, at which crystals grow quickly and, in the case of no crystal seeds feeding, nucleation can occur to supply proper number of nucleuses [57]. LIS promotes micromixing very efficiently and so can provide such conditions. Also, it has the feature of considerably strong pressure fluctuation, which, together with efficient micromixing, enhances process kinetics, as described in Section 3.4. So, crystallization is also an occasion where LIS is especially applicable. Based on the fundamental investigations the nonrotating flow impinging stream vacuum crystallizer (NRFISVC) and the nonrotating flow impinging stream heat-exchanging crystallizer (NRFISHEC) were developed by $\mathrm{Wu}[58,59]$, and the former is shown in Figure 13. The basic working principles of NRFISVC are somewhat similar to those of the will known DTB crystallizer; while its mechanical structure and flow configuration inside the container are essentially the same as the no-rotating flow vertical circulative impinging stream reactor employing LIS, except to the classifying leg and flow in it. The impingement of opposing streams against each other provides well micromixing and, consequentially, uniform supersaturation; while the average supersaturation requested can be easily achieved by controlling the vacuum degree, consequentially, the temperature of the crystals-inmother liquor suspension, and the feed concentration. At the bottom of the container a classifying leg is placed, and the recycled mother liquor drawn from the overflow port enters the leg and flow upward to create a certain upwardvelocity so that smaller crystals are carried upward back into the container to continue growth; while those large enough drop down by gravity and then, with a part of mother liquor, are discharged from the bottom of the leg and are delivered to the separation machine, normally centrifuge(s).

Since 2007, the nonrotating flow impinging stream vacuum and heat-exchanging crystallizers has been applied industrially; now, totally 23 sets of large scale, 18 sets of effective volume $32 \mathrm{~m}^{3}$, and others of $20 \mathrm{~m}^{3}$ are in operation in three companies in China for production of various products, and all they are working well.

In addition, recently the reactor shown in Figure 10 was used for extraction in a pilot plant test for purification of phosphoric acid and exhibited very well performance, as predicted by $\mathrm{Wu}$ in $[4,5]$, and the detailed results will be reported elsewhere.

It is trusted that, in addition to those described above, impinging streams can find more and more applications because of their excellent features.

\section{Concluding Remarks}

Impinging streams (IS) are classified into two categories: gascontinuous impinging streams (GIS) and liquid-continuous impinging streams (LIS). They have individual and quite different features and thus are applicable for various occasions. All the features of IS, including GIS and LIS, have great effects on enhancing processes. One very important thing in application is that the target system has to be chosen properly according to the properties of both the specific IS and the target system.

The major feature of GIS is enhancing efficiently transfer between phases; while it has the intrinsic disadvantage of very short residence time. So, GIS alone is applicable especially and only for rapid or instant processes controlled by diffusion through gas film. Except to combustion, the most important application of GIS may be for wet FGD with dehydrated lime as the absorbent; an equipment system employing GIS of quasiindustrial scale can be expected to be built in the near future.

The effect of LIS enhancing transfer is negligible; while, due to strong interaction between the opposing streams, it has the important features of efficient micromixing and considerably strong pressure fluctuation favoring process kinetics and so is suitable for processes occurring on molecular scale in liquid, especially those involving reaction(s), and its application field should be much wider than GIS for such processes are widely involved in many process industries. Results of number investigations have shown that preparation of ultrafine materials by reaction precipitation is a potential area of LIS application, and that improving reaction device is an effective way to enhancing technologies for preparation of nano materials, too. Since 2007, a number of reactors and crystallizers employing LIS, NRFVCISR, NRFISVC and NRFISHEC, of large scales, have been, as the first time, successfully applied in industry, and operated for long term without any faultiness. Because of its high efficiency, great energy saving, and the special functions in producing finer nanomaterials and enhancing selectivity of reactions, and so forth, NRVCISR can be expected to be the ideal substitute of the traditional stirred tank reactor (STR).

It is trusted that, in addition to those described here, impinging streams can find more and more applications in various processing industries because of their excellent features.

\section{Nomenclature}

a: $\quad$ Specific area, $\mathrm{m}^{2} \cdot \mathrm{m}^{-3}$

$\mathrm{Ca} / \mathrm{P}$ : Mole ratio of calcium to phosphorus

$\mathrm{Ca} / \mathrm{S}$ : Mole flow rate ratio of calcium to sulfur

$D_{a}$ : Diameter of absorption chamber, $\mathrm{m}$

$d_{0}$ : Diameter of gas flow conduit, $\mathrm{m}$ 
$u_{0}$ : Impinging velocity, $\mathrm{m} \cdot \mathrm{s}^{-1}$

$H_{a}$ : Height of absorption chamber, $\mathrm{m}$

$h$ : Height of gas conduit from the lower edge of the cylinder chamber, $\mathrm{m}$

$K_{\mathrm{IS}}$ : Overall crystal-growth rate coefficient in IS crystallizer, $\mathrm{m} \cdot \mathrm{s}^{-1}$

$K_{\mathrm{FB}}$ : Overall crystal-growth rate coefficient in fluidized-bed crystallizer, $\mathrm{m} \cdot \mathrm{s}^{-1}$

$k_{G}$ : Gas-film mass transfer coefficient, $\mathrm{m} \cdot \mathrm{s}^{-1}$

$K_{L}$ : Liquid-film mass transfer coefficient, $\mathrm{m} \cdot \mathrm{s}^{-1}$

$k_{\mathrm{IS}}$ : Reaction rate constant in IS device, $\mathrm{m}^{3} \mathrm{kmol}^{-1} \mathrm{~s}^{-1}$

$k_{\mathrm{STR}}$ : Reaction rate constant in stirred tank reactor, $\mathrm{m}^{3} \cdot \mathrm{kmol}^{-1} \cdot \mathrm{s}^{-1}$

$S: \quad$ Impinging distance, $m$

$t_{M}$ : Micromixing time, milliseconds

$\lambda$ : Kolmogoroff microscale.

\section{Acknowledgment}

The projects supported by The National Natural Science Foundation of China (No. 29276260, 20176043, and 20424002).

\section{References}

[1] I. T. Elperin, "Heat and mass transfer in opposing currents," Journal of Engineering Physics, vol. 6, pp. 62-68, 1961 (Russian).

[2] A. Tamir, Impinging Stream Reaction-Fundamentals and Application, Elsevier, Amsterdam, The Netherlands, 1994.

[3] Y. Wu, "Diversion of the emphasis in investigation on the continuous phase in impinging streams," Chemical Industry and Engineering Progress, vol. 22, no. 10, pp. 1066-1071, 2003 (Chinese).

[4] Y. Wu, Impinging Streams-Fundamentals, Properties, and Applications, Elsevier, Amsterdam, The Netherlands, 2007.

[5] Y. Wu, "Properties and application of impinging streams," Chemical Industry and Engineering Progress, vol. 20, no. 11, pp. 8-13, 2001 (Chinese).

[6] Y. Wu, "A rustic opinion for the definition of impinging streams," Huaxue Gongcheng, vol. 36, no. 9, 2008 (Chinese).

[7] Y. Wu, "Properties and application of impinging streams," Chemical Industry \& Engineering Progress, vol. 20, no. 11, pp. 8-13, 2001 (Chinese).

[8] K. Huang, H. Liu, and Y. Wu, "A study on circulative impinging stream drying," Chemical Engineering Journal of Colleges and Universities of China, vol. 15, no. 3, pp. 78-80, 2001 (Chinese).

[9] Y. Wu, Q. Li, and F. Li, "Desulfurization in the gas-continuous impinging stream gas-liquid reactor," Chemical Engineering Science, vol. 62, no. 6, pp. 1814-1824, 2007.

[10] R. H. Perry and D. Green, Chemical Engineer's Handbook, McGraw-Hill, New York, NY, USA, 6th edition, 1984.

[11] A. Tamir and A. Glizenstien, "Modeling and application of a Semibatch coaxial two-impinging-stream contactor for dissolution of solids," The Canadian Journal of Chemical Engineering, vol. 70, pp. 104-114, 1992.

[12] A. Tamir and M. Grinholtz, "Performance of a continuous solid-liquid two-impinging-stream reactor," Industrial and Engineering Chemistry Research, vol. 26, no. 4, pp. 726-731, 1987.
[13] J. T. Davies, "Particle suspension and mass transfer rates in agitated vessels," Chemical Engineering Progress, vol. 8, pp. 175-181, 1986.

[14] Y. Wu, Y. Xiao, and Y. Zhou, "Micromixing in the submerged circulative impinging stream reactor," Chinese Journal of Chemical Engineering, vol. 11, no. 4, pp. 420-425, 2003.

[15] Y. Wu, "The submerged circulative impinging stream reactor," CNP ZL00 2 30326.4, 2000.

[16] A. J. Mahajan and D. J. Kirwan, "Micromixing effects in a twoimpinging-jets precipitator," AIChE Journal, vol. 42, no. 7, pp. 1801-1814, 1996.

[17] J. Zhang, Y. Wu, A. Shu, and C. Xu, "Characteristics of pressure fluctuation in submerged circulative impinging stream reactor," Journal of Chemical Industry and Engineering, vol. 56, no. 2, pp. 266-269, 2005 (Chinese).

[18] H. Sun, Y. Wu, and C. Xu, "Pressure fluctuation in the submerged circulative impinging stream reactor," Chinese Journal of Chemical Engineering, vol. 14, no. 4, pp. 428-434, 2006.

[19] Y. Wu, Y. Zhou, and C. Bao, "Features of liquid-continuous impinging streams and their influences on kinetics," in New Developments and Application in Chemical Reaction Engineering, H.-K. Rhee, I.-S. Nam, and J. M. Park, Eds., vol. 159 of Studies in Surface Science and Catalysis, pp. 533-536, Elsevier, Amsterdam, The Netherlands, 2006.

[20] Y. Zhou, Y. Wu, and C. Bao, "Crystal growth rate of Na2HPO4 in impinging stream crystallizer," Huaxue Gongcheng, vol. 35, no. 12, pp. 12-17, 2007 (Chinese).

[21] B. Chen, C. Bao, and Y. Wu, "A study on reaction kinetics in impinging stream reactor," Henan Chemical Industry, vol. 10, 2006.

[22] Y. Wu and Y. Zhou, "The vertical circulative impinging stream reactor," CNP ZL2005 2 0094812.2, 2005.

[23] Y. Wu and X. X. Wu, "The non-rotating flow vertical circulative impinging stream reactor," CNP ZL2007 2 0083472.3, 2007.

[24] G. J. Pitt and G. R. Millward, Coal and Modern Coal Processing: An Introduction, Academic Press, New York, NY, USA, 1979.

[25] H. Staege, "The production of methanol and hydrogen by Kopper-Totzek coal gasification process," Erdöl-ErdgasZeitschrift, vol. 92, pp. 381-387, 1976.

[26] S. Munjal and P. Dudukovic, "Mass-transfer in rotating packed beds-II. Experimental results and comparison with theory and gravity flow," Chemical Engineering Science, vol. 44, no. 10, pp. 2257-2268, 1989.

[27] F. Li, An investigation into wet desulfurization of flue gas by impinging stream absorption, M.S. thesis, Wuhan Institute of Technology, Wuhan, China, 2005.

[28] Y. Berman, A. Tanklevsky, Y. Oren, and A. Tamir, "Modeling and experimental studies of $\mathrm{SO}_{2}$ absorption in coaxial cylinders with impinging streams: part I," Chemical Engineering Science, vol. 55, no. 5, pp. 1009-1023, 2000.

[29] Y. Wu, C. Bao, and Y. Zhou, "The impinging stream gas-liquid reactor," CNP ZL2004 2 0017226.4, 2004.

[30] Y. Wu, G. Wu, and Y. Zhou, “The eddy pressure nozzle," CNP ZL00230305.1, 2000.

[31] H. Chen, X. Deng, J. Zhang, and J. Zhang, "Measurement of the effective interface area and volumetric mass transfer coefficient in multistage rotating packed bed with centrifugal atomizing by chemical absorption," Chemical Reaction Engineering \& Processing, vol. 15, no. 1, pp. 97-103, 1999 (Chinese). 
[32] H. Zhao, Investigation on hydrodynamics and mass transfer performance of multistage cross-flow rotating packed bed, M.S. thesis, Central North University, Taiyuan, China, 2004.

[33] S. Munjal and P. Dudukovic, "Mass-transfer in rotating packed beds-I. Development of gas-liquid and liquid-solid masstransfer correlations," Chemical Engineering Science, vol. 44, no. 10, pp. 2245-2256, 1989.

[34] Z. Chen, S. Xiong, and J. Wu, "Rotating absorber of screw type," Journal of Chemical Industry and Engineering, vol. 46, no. 3, pp. 388-391, 1995 (Chinese).

[35] Y. Wu, X. X. Wu, and X. H. Wu, "The huge flowrate impinging stream gas-liquid reactor," CNP ZL200620095838.4, 2006.

[36] Y. Wu and X. H. Wu, "The large flowrate eddy pressure nozzle," CNP ZL2006 2 0099102.4, 2006.

[37] Y. Zhou, T. Yang, J. Guo, and Y. Wu, "Preparation of nano zinc oxide by impinging stream reaction-precipitation," Huaxue Gongcheng, vol. 36, no. 5, pp. 55-58, 2008.

[38] Y. Wu, Y. Xiao, and Y. Zhou, "Submerged circulative impinging stream reactor," Chemical Journal on Internet, vol. 4, no. 9, p. 44, 2002.

[39] Y. Wu and Y. Chen, "Preparation of "ultra-fine" white carbon black in submerged circulative impinging stream reactor," Journal of Chemical Industry and Engineering, vol. 54, no. 10, pp. 1381-1386, 2003 (Chinese).

[40] Z. Chen, Preparation of nano copper powder by impinging stream reaction-precipitation, M.S. thesis, Wuhan Institute of Technology, Wuhan, China, 2004.

[41] Z. Liu, B. Zhao, Z. Zhang, and L. Hu, "Preparation of super micro copper powder with formaldehyde as the reducing agent," Chemistry, no. 11, pp. 25-26, 1996 (Chinese).

[42] Z. Liu, B. Zhao, Z. Zhang, and L. Hu, "Preparation of super micro copper powder with Ascorbic acid as the reducing agent and thermo- stability of the product," Journal of Eastern China University of Technology, vol. 22, no. 5, pp. 548-553, 1996 (Chinese).

[43] B. Zhao, Z. Liu, M. Cai, and L. Hu, "A study on the preparation of ultrafine copper powder by hydrazine hydrate reducing and stability of the product," Journal of Eastern China University of Technology, vol. 23, no. 3, pp. 372-376, 1997 (Chinese).

[44] Z. Zhang, H. Han, and M. Sun, "Preparation of nano copper powder," Fine Chemicals Industry, vol. 17, no. 2, pp. 69-71, 2000 (Chinese).

[45] H. Zhang, S. Bai, X. Zhao, and F. Tao, "Preparation of nano copper powder by chemical reduction," Materials in Mechanical Engineering, vol. 22, no. 3, pp. 33-34, 1998 (Chinese).

[46] W. Yuan and X. Jin, "Methods for preparation of nano copper powder," CNP app. no. 97100501.X, 1997.

[47] Z. Guo, K. Gu, and J. Xu, "Lubricating and self-repairing effects of nano Lubricating additive," Material Protection, vol. 36, no. 9, pp. 21-23, 2003 (Chinese).

[48] Z. Liu, B. Zhao, and L. Hu, "Surface improvement of copper micro powder and its anti-oxidation nature," Journal of Eastern China University of Technology, vol. 22, no. 3, pp. 270275, 1996 (Chinese).

[49] B. Zhao, Z. Liu, M. Cai, and L. Hui, "An investigation on preparation of ultrafine copper powder by reduction with hydrazinium and its stability," Journal of Eastern China University of Technology, vol. 23, no. 3, pp. 372-376, 1997 (Chinese).

[50] B. Gao, J. Gao, H. Jiang, and Z. Zhang, "Cladding structure of nano copper-silver double metal powder and its antioxidation property," Journal of Physical Chemistry, vol. 16, no. 4, pp. 366-369, 2000 (Chinese).
[51] G. Li, Preparation of nano Titania by impinging stream reaction-precipitation, M.S. thesis, Wuhan Institute of Technology, Wuhan, China, 2003.

[52] J. Yuan, Y. Wu, and Q. Zheng, "Synthesis of nano rods/whiskers of hydroxyapatite by precipitation in impinging streams," in Proceedings of the 11th Symposium of Asian Pacific Confederation of Chemical Engineering, Kuala Lumpur, Malaysia, 2006.

[53] Zhou, et al., "Preparation of nano magnesium oxide by impinging stream reaction," Henan Chemical Industry, vol. 24, no. 4, pp. 22-24, 2007 (Chinese).

[54] Y. Zhou, J. Liu, D. Mi, and Y. Wu, "Preparation of nano barium titanate by impinging stream reaction," Inorganic Salts Industry, no. 9, pp. 15-17, 2007 (Chinese).

[55] Y. Zhou, J. Zhang, Z. Li, J. Guo, and Y. Wu, "Preparation of nano strontium titanate by reaction-precipitation," Chemical Minerals and Processing, vol. 37, no. 7, pp. 4-6, 2008.

[56] Y. Wu and X. X. Wu, "The non-rotating flow vertical circulative impinging stream reactor,” CNP CNP ZL200720083472.3, 2007.

[57] X. Ding and Y. Tan, Industrial Crystallization, Chemical Industry Press, Beijing, China, 1985.

[58] Y. Wu, "Non-rotating flow impinging stream vacuum crystallizer," CNP ZL 20072 0083842.3, 2007.

[59] Y. Wu, "Non-rotating flow impinging stream heat-exchanging crystallizer,” CNP ZL 200820066734.9, 2008. 

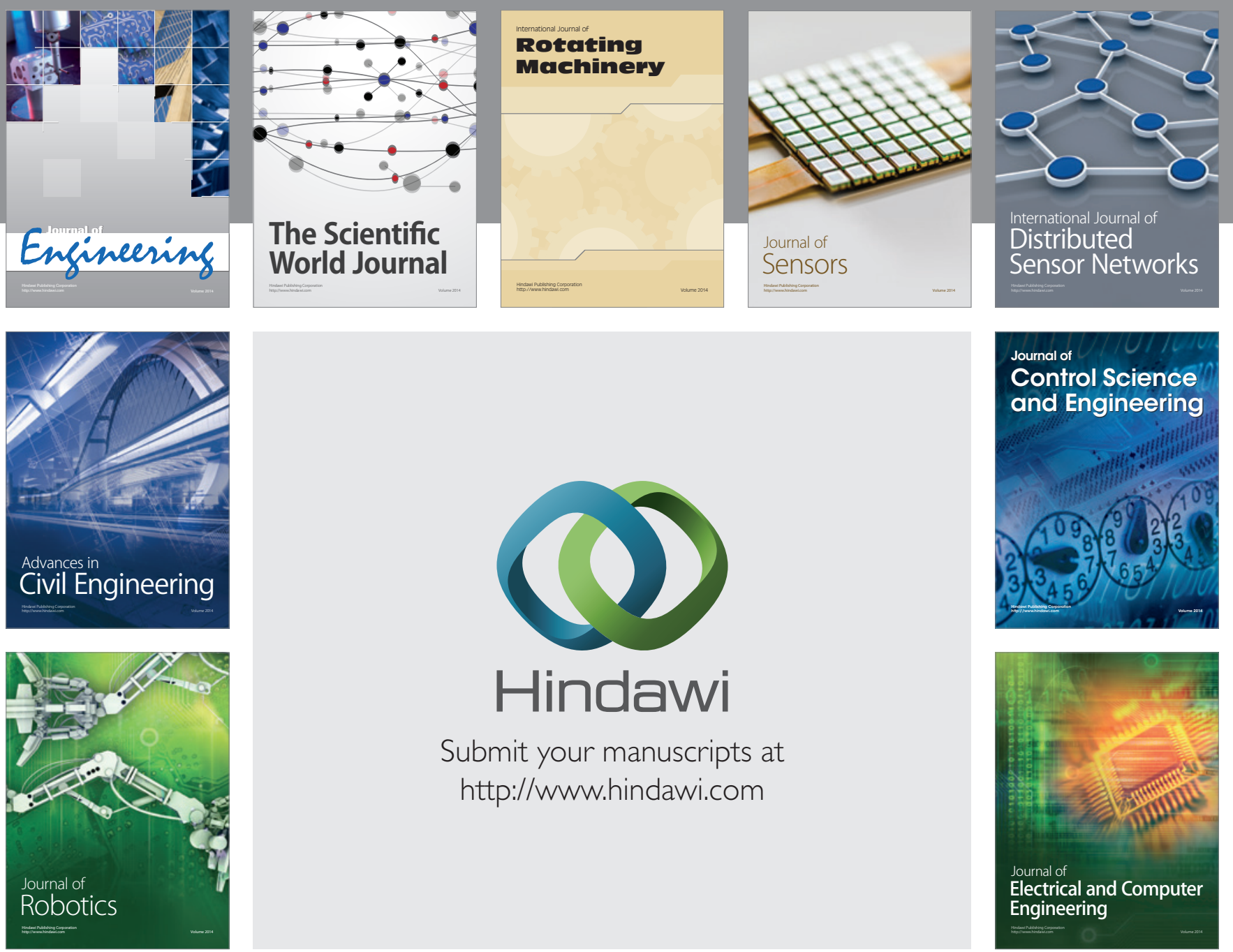

Submit your manuscripts at

http://www.hindawi.com
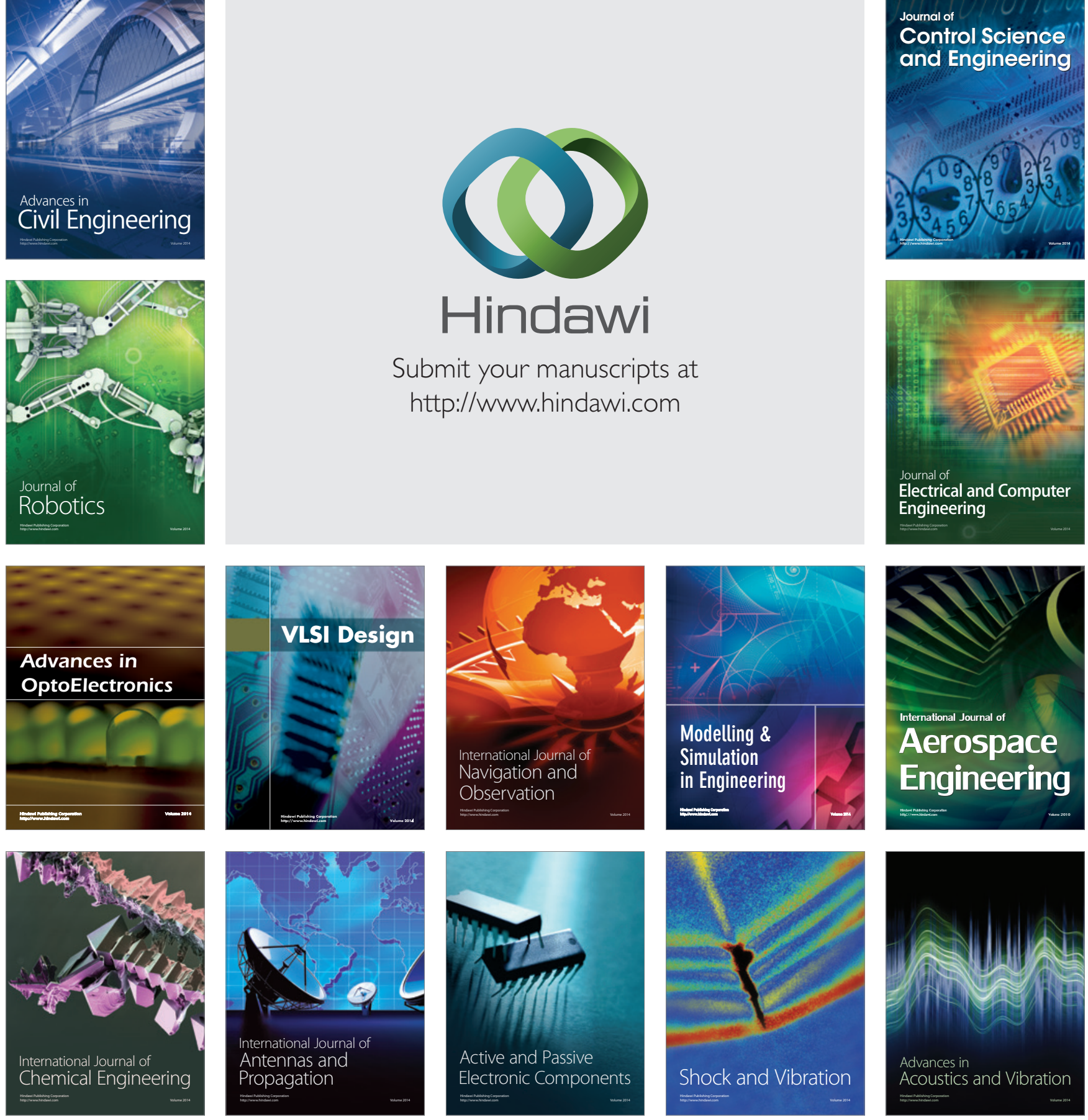